valente en otras obras del artista, como El asilo de San Bernardino de Madrid (Museo Municipal. Madrid) o la Vista de la Casa de Campo (óleo/lienzo de 0,50 × 0,69 m. subastado en Durán en febrero de 2002, lote 222), por citar algún ejemplo. Y lo mismo podemos decir de la factura de los árboles, con sus característicos y esbeltos troncos, realizados a base de largas rayas sobre el empaste, patente en cuadros como Vichy (Fig. 10) ${ }^{17}$ (óleo/cartón, 0,255 x 0,34 m., colección particular. Madrid), de gran semejanza con los alargados y rayados troncos del cuadro que estudiamos; pero no sólo son los troncos, sino también la factura de las copas de los árboles las que se convierten en otro referente de identidad si las comparamos, por ejemplo, con las representadas en cuadros como Vista de Toledo (Museo de Arte Moderno. Barcelona), de hojarasca de factura tan igual con la del cuadro que estudiamos; o si nos fijamos en la forma tan peculiar de terminar las copas de los árboles en cuadros como Vista del sur de Toledo desde los Cigarrales (Museo de Arte Contemporáneo. Toledo) o la Vista de la Vega Baja desde El Cambrón: el río y sus riberas con la fábrica de armas al fondo, del mismo Museo, o Paisaje de Segovia (Fig. 11) (óleo/lienzo, 0,40 × 0,60 m., Colección de Arte de Caja Madrid), tan semejantes a la manera como se rematan las copas de los árboles en este cuadro que presentamos. Todo ello, como podemos ver, son elementos comunes de identidad, presentes tanto en este cuadro como en esas y otras obras de Aureliano de Beruete.

ENRIQUe ARIAS ANGLÉS I. Historia (CSIC)

\title{
EL RETRATO DE LA ÚLTIMA HIJA DE JUAN BAUTISTA MARTÍNEZ DEL MAZO, BOCETO PARA EL RETRATO DE LA FAMILIA DEL PINTOR DEL KUNSTHISTORISCHES MUSEUM DE VIENA
}

Por adquisición en el comercio de antigüedades francés se conserva en una colección particular de Bilbao el Retrato de una niña (fig. 2), pintura de carácter velazqueño, que puede ser clasificada perfectamente como obra indiscutible del pintor Juan Bautista Martínez del Mazo (¿Beteta?, Cuenca, 1611-Madrid, 1667), tanto por la característica soltura técnica, como por tratarse de uno de los modelos infantiles que aparecen en el Retrato de la familia del pintor del Kunsthistorisches Museum de Viena (fig. 3), en relación con la cual se conoce otro Retrato de Luis Martínez del Mazo conservado en la Dulwich Picture Gallery de Londres (fig. 1) ${ }^{1}$. La importancia de la obra es notable no sólo porque incrementa el catálogo de Martínez del Mazo con una obra indudable, sino también por contribuir a precisar la cronología de la pintura conservada en Viena ${ }^{2}$.

\footnotetext{
${ }^{17}$ Ibidem, pp. 422-423.

${ }^{1}$ Esta pintura ya aparece recogida en la segunda edición de 1903 de la obra Carl Justi, Velázquez y su siglo. Madrid, 1953, p. 774. Se reproduce en el Velázquez de los Klassiker der Kunst, Stuttgard y Berlín, 1913, p. 229. Juan Antonio Gaya Nuño. La pintura española fuera de España. Historia y catálogo. Madrid, 1958, p. 230, $\mathrm{n}^{\circ} 1727$. Su reproducción puede verse en el libro de José López-Rey Velázquez, Painter of painters. Colonia, Taschen - Wildenstein Institute, 1996, vol. I, p. 190. El niño retratado en el lienzo de Londres puede ser identificado con don Luis del Mazo y de la Vega, segundo hijo del matrimonio del pintor con Francisca de la Vega (Vid. infra). Así se recoge en la obra de Richard Beresford, Dulwich Picture Gallery. Completed Illustrated Catalogue. Londres, 1998, p. 157.

${ }^{2}$ Bibliografía básica sobre el pintor que se citará en las notas es la siguiente: $\mathrm{M}^{\mathrm{a}}$ Luisa Caturla, «Sobre un viaje inédito de Mazo a Italia, hasta ahora ignorado», en Archivo Español de Arte, 1955, pp- 7-75. José López Navío. «El matrimonio de Juan Bautista Martínez del Mazo con la hija de Velázquez», en Archivo Español de Arte, xxxiI, 1960, pp. 389-419. Varia velazqueña. Homenaje a Velázquez en el III Centenario de su muerte. 1660-1960. Volumen II. Elogios poéticos. Textos y comentarios críticos. Documentos. Cronología. Láminas e índices. Madrid, 1960. Desde el documento 209 en adelante. Juan Antonio Gaya Nuño, «Juan Bautista del Mazo el gran discípulo de Velázquez», en Varia Velazqueña. Home-
} 
Representa el busto de una niña de corta edad, como de un año o poco más, ligeramente girada hacia su lado derecho, que es el mismo que adopta en la composición definitiva de la pintura de Viena. Lleva un vestido de color pardo rojizo liso, que cambia a azulado en el lienzo definitivo, sin más adornos que los cuellos blancos que orlan el escote y el broche central sobre el pecho. La niña luce un peinado indefinido de melena corta y rizada, y un pendiente colgante sobre la oreja visible. Su intensa expresión resulta mitad enfadada, mitad perdida, como si hubiera fijado la mirada en algún punto fuera del espacio del lienzo.

Se trata de una pintura al óleo sobre lienzo que mide $38 \times 27$ centímetros. El estado de conservación es muy bueno. La radiografía de la obra muestra un trazo limpio y certero en la figura, abordada por el pintor directamente y sin arrepentimientos, si bien delata una leve pérdida de pintura en el fragmento de la tela del cuello izquierdo, junto al broche que separa las dos mitades, la cual no afecta a ninguna parte sustancial de la obra.

La pintura está forrada desde antiguo, quizá desde fines del siglo XVIII, sobre una tela ligeramente más amplia ${ }^{3}$ y dispuesta sobre un bastidor en cuyo travesaño central, hacia su mitad izquierda, puede leerse «Diego Velazquez», con falta de alguna letra en el nombre del pintor, lo cual supone una bien centrada clasificación antigua de la obra en torno al gran maestro de la pintura barroca española, con independencia de que quien la hiciera conociera o no el cuadro de Martínez del Mazo del Kunsthistorisches Museum de Viena.

Desde un punto de vista técnico, la pincelada es fluida, especialmente ligera sobre el colorido pardo del vestido que parece aprovechar la imprimación de la base, cuya oscuridad se enciende por contraste con la blancura y la hechura pastosa de los cuellos a base de pinceladas espumosas y rizadas. En el rostro los matices son más cuidadosos, acentuando los carmines sobre las mejillas, las luces sobre los pómulos y la nariz, y la intensa mirada de los ojos oscuros. Mazo demuestra en esta obra sus buenas dotes para el retrato infantil, en el que tanto colaboró con Velázquez en los últimos años de su vida.

Un problema que surge al considerar este retrato en relación con la obra maestra de Martínez del Mazo es a quién representa y si se trata de un boceto preparatorio para el Retrato de la familia del pintor de Viena o más bien de una obra independiente.

¿Quién es el personaje retratado? Para saberlo con la mayor precisión posible, conviene remarcar la naturaleza del Retrato de la familia de Martínez del Mazo. Es un retrato colectivo de los distintos componentes de la familia del pintor, que se retrató al fondo ejecutando un retrato de la infanta Margarita. Mazo había contraído matrimonio en 1634 con Francisca Velázquez, hija de su maestro Diego Velázquez, la cual había fallecido a finales de 1653. Al año siguiente la hija mayor de esta unión, doña Inés, contrajo matrimonio con el napolitano Onofre de Lifrangis, trasladándose a vivir a Nápoles, aunque en 1661 volvía a contraer matrimonio en Madrid con don Pedro Rodríguez Cortés ${ }^{4}$. En 1655, los demás hijos del primer matrimonio, salvo Gaspar, eran todos menores de edad: Baltasar, menor de catorce años, y Teresa y Melchor, menores de siete años.

naje a Velázquez en el III Centenario de su muerte. 1660-1960,Volumen I. Estudios sobre Velázquez y su obra. Madrid, 1960, pp. 471-481. Elizabeth Du Gue Trapier, «Martínez del Mazo as a Landscapist», en Gazette des Beaux Arts, 61, 1963, pp. 292-310. Mercedes Agulló Cobo, Noticias sobre pintores madrileños de los siglos XVI y XVII. Granada, 1978, pp. 9596. Id., Más noticias sobre pintores madrileños de los siglos xVI al xVIII. Madrid, 1981, pp. 134-136. Peter Cherry, «Juan Bautista Martínez del Mazo, viudo de Francisca Velázquez», en Archivo Español de Arte, LXIII, 1990 , pp. 511-527. Mercedes Agulló Cobo, Documentos para la historia de la Pintura española. I. Madrid, 1994, pp. 68-72. Corpus velazqueño. Documentos y textos. Madrid, 2000.

${ }^{3}$ El Retrato de Luis del Mazo de la Dullwich Picture Gallery mide prácticamente lo mismo: 37,8 × 27,1 centímetros (Beresford, op. cit., 1998, p. 157). La medida de $53 \times 26 \mathrm{~cm}$ proporcionada por Gaya Nuño está equivocada (op. cit., 1958, p. 230, $\mathrm{n}^{\circ} 1727$ ).

${ }^{4}$ Varia Velázqueña. Volumen II. Madrid, 1960, p. 402, documento 213. Se celebró el matrimonio el 6 de febrero de 1661.

AEA, LXXVIII, 2005, 311, pp. 297 a 331 

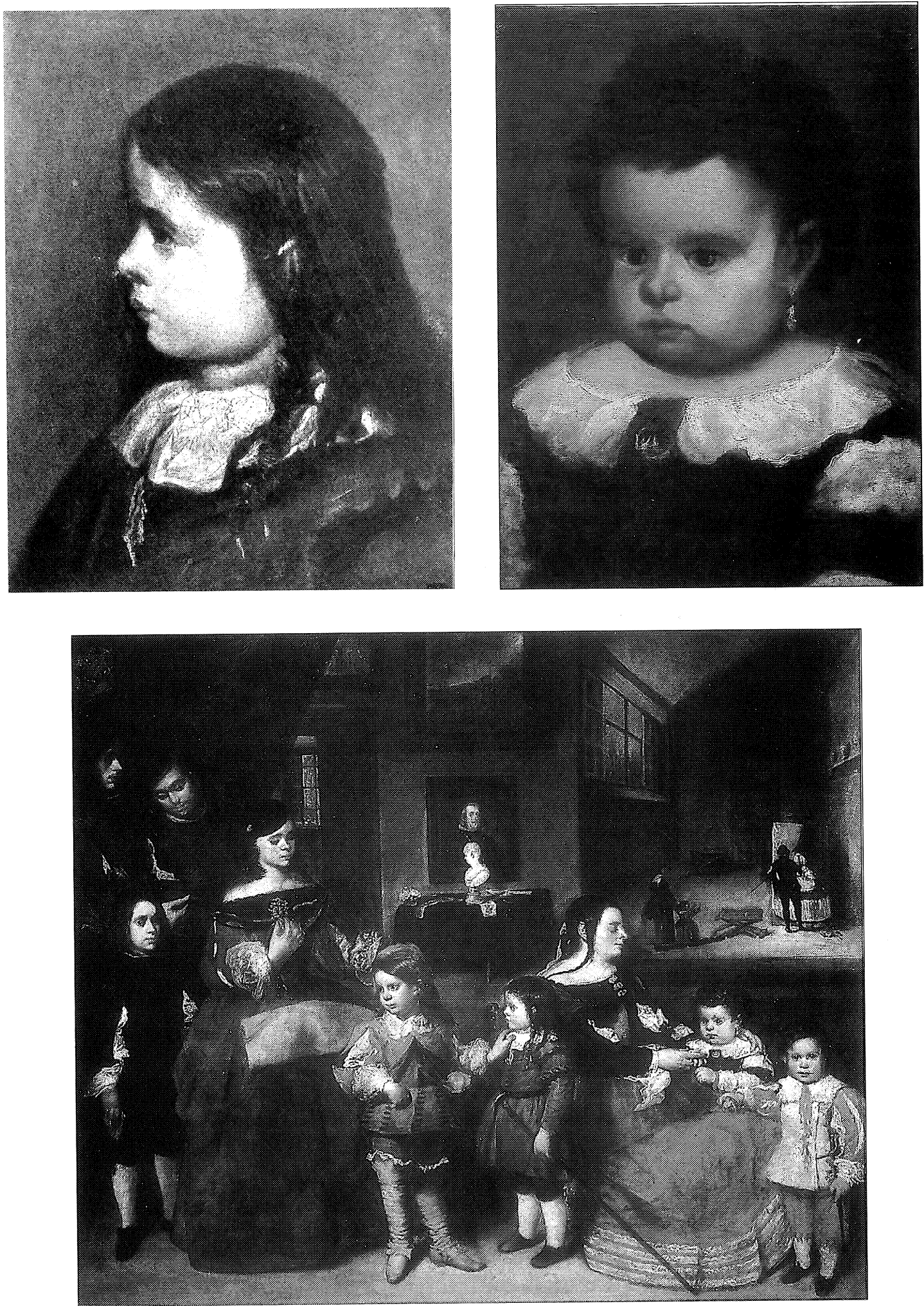

Fig. 1. Retrato de Luis Martínez del Mazo (Londres, Dulwich Picture Gallery)

Fig. 2. Retrato de una niña (Bilbao, colección particular).

Fig. 3. Retrato de la familia del pintor (Viena, Kunsthistorisches Museum).

AEA, LXXVIII, 2005, 311, pp. 297 a 331 
Después de 1654 Mazo contrajo segundo matrimonio con Francisca de la Vega, fallecida e1 22 de marzo de $1665^{5}$.

Poco después contraería tercer matrimonio con Ana de la Vega, a quien se ha supuesto hermana de la anterior, que sobrevivió al pintor ${ }^{6}$, fallecido el 10 de febrero de 1667 . Ana de la Vega aparece casada posteriormente con don Alonso Carrillo cuando declaraba el 4 de octubre de 1668 que había recibido cierta cantidad de dinero del alquiler de unas casas de aposento que correspondían a los menores que tenía a su cargo, a saber «don Juan, don Luis y don Francisco del Mazo y de la Vega, hijos y herederos de don Juan Bauptista Martínez del Mazo, pintor de Cámara y ayudante de la Furriera que fue de Su Magestad» ${ }^{7}$. Sin duda alguna se trata de los hijos del segundo matrimonio, los tres niños mayores que aparecen en el retrato de Viena alrededor de la mujer sentada, pero que no mantienen una especial relación de afectividad con ella, como tampoco la tienen los cuatro jóvenes del lado izquierdo, que sin duda son los hijos del primer matrimonio con Francisca Velázquez. Al saberse que en 1668 Ana de la Vega estaba al cargo de la «educación y alimentos» de tres menores del anterior matrimonio de su esposo ${ }^{8}$, hay que suponer que el cuarto niño del retrato de Viena (en realidad una niña), el que se apoya en la mujer, en parte por ser su madre y en parte por su corta edad que apenas le permitiría mantenerse en pie, es el último de los hijos de Mazo y de Ana de la Vega, cuyo nombre desconocemos. La catalogación del Retrato de la familia del pintor (Viena, Kunsthistorisches Museum) en la reciente exposición sobre El retrato español, reconociendo la dificultad para identificar a todos los personajes, precisa algunos datos biográficos sobre los mismos $\mathrm{y}$, sin tener en cuenta el tercer matrimonio de Mazo, deshace antiguas confusiones e identificaciones y puntualiza que el menor de los chicos es en realidad una niña ${ }^{9}$, lo cual imposibilita que se trate de una hija de Francisca de la Vega, y refuerza nuestra hipótesis de que se trate de la última hija del pintor, habida en el tercer matrimonio con Ana de la Vega. Nuestra hipótesis permitiría datar éste retrato entre 1666 y $1667^{10}$, al igual que la obra definitiva del museo de Viena, que Mena Marqués data entre 1664-1665 ${ }^{11}$, mejor que en la difusa fecha de 16601665 en que ha sido situado tradicionalmente, por considerar que el pintor del fondo es el mismo Velázquez, fallecido en $1660^{12}$.

Sin embargo, parece más lógico considerar que este pintor que aparece en la estancia-taller del fondo es el propio Juan Bautista Martínez del Mazo, pintando uno de los retratos de la infanta Margarita de Borbón, según el modelo que se conserva en el Museo del Prado ${ }^{13}$, teni-

\footnotetext{
${ }^{5}$ Varia Velazqueña, volumen II, 1960, p. 404, documento 220. Corpus Velazqueño. Textos y documentos, II, Madrid, $2000, \mathrm{n}^{\circ} 463$, p. 517.

${ }^{6}$ Juan Antonio Gaya Nuño. «Juan Bautista Martínez del Mazo, el gran discípulo de Velázquez», en Varia Velazqueña, Madrid, 1960, pp. 472

${ }^{7}$ Mercedes Agulló y Cobo. Noticias sobre pintores madrileños de los siglos XVI y XVII. Granada, 1978. p. 95. Un niño llamado Fernando, hijo de Mazo y de Francisca de la Vega fue bautizado el 7 de noviembre de 1663 en la parroquia de San Juan de Madrid, siendo apadrinado por don Juan Bautista del Mazo «su hermano» y por Ana de la Vega (Corpus Velazqueño. Documentos y textos, II. Madrid, 2000, $\left.\mathrm{n}^{\circ} 459, \mathrm{p} .515\right)$. Pero no se le menciona como heredero de su madre en la partida de entierro del 22 de marzo de 1665, por lo que fallecería en ese intervalo de tiempo (op. cit., $\mathrm{n}^{\circ}$ 464, p. 517)

${ }^{8}$ Se ha sugerido que Ana de la Vega pudiera haber sido hermana de Francisca de la Vega. La rapidez en contraer matrimonio con otra mujer del mismo apellido da pie a creerlo con cierto fundamento, pero el documento citado de octubre de 1668 no menciona ni que las mujeres fueran hermanas, ni que los niños que educaba fueran sus sobrinos (Gaya Nuño, op. cit., 1960, p. 472).

${ }^{9}$ Catálogo de la exposición El retrato español, del Greco a Picasso. Madrid, Museo Nacional del Prado, 2004, p 351, $\mathrm{n}^{\circ} 44$, con texto de MMM(Manuela Mena Marqués).

${ }^{10}$ López-Rey considera que el retrato de la Dulwich Picture Gallery es obra de hacia 1660-1661 (op. cit., 1996, vol. I, p. 190), lo cual no parece posible.

${ }^{11}$ Ibidem.

${ }^{12}$ Para un análisis de los pros y contras en la identificación de los personajes véase Justi, 1953, pp. 772-776. Gaya Nuño, op. cit., 1960 , p. 479.

${ }^{13}$ Cherry, op. cit., 1990 , p. 516
}

$A E A$, LXXVIII, 2005, 311, pp. 297 a 331 
do tradicionalmente como obra de Velázquez, pero adjudicado en la actualidad sin reservas al propio Mazo ${ }^{14}$. Consiguientemente la mujer sentada sería Ana de la Vega con su hija, la última del pintor nacida del tercer matrimonio. No cabe duda de que el retrato familiar de Mazo es un homenaje a los modos pictóricos y conceptuales de su suegro Diego Velázquez y desde el punto de vista de los personajes representados una síntesis de las familias que formó el pintor, pues aparecen los hijos de sus tres matrimonios, compendiadas en los últimos años de su vida.

Respecto a la pintura que nos ocupa, por el momento no es posible reconstruir su historia anterior a su adquisición en Francia en una subasta pública. Parece lógico pensar que a la muerte de Martínez del Mazo quedara entre los bienes y utensilios del taller. Pero por el momento no se conoce la existencia de ningún testamento ni inventario de los bienes del pintor en el que se pueda rastrear la presencia de éste retrato y de otros del mismo tipo que parece que el pintor realizó a sus hijos como estudios previos para el gran cuadro de Viena, como lo demuestra el lienzo de la Dulwich Picture Gallery.

Dr. ISMAel GutiérRez PASTOR Universidad Autónoma de Madrid

\section{NUEVAS ESCULTURAS DE LUIS Y JOSÉ SALVADOR CARMONA ?*}

En la villa ducal de Béjar es, especialmente, venerada una imagen de la Piedad que, procedente del desaparecido monasterio franciscano, recibe hoy culto en la iglesia de Santa María la Mayor (figs. 1 y 2). La imagen procesiona durante la Semana Santa y allí se la tiene, en general, como obra del escultor salmantino Alejandro Carnicero.

La escultura, muy hermosa, no encaja con lo conocido de Carnicero. Precisamente este escultor tallaba el mismo tema para la hornacina superior del retablo de la catedral de Coria, en Cáceres, terminado en 1748 , y su estilo, más dramático y con un tratamiento de paños más minucioso y movido, es distinto al de la obra que nos ocupa ${ }^{1}$. En la Piedad de Béjar, lo que llama especialmente la atención es su serenidad y su sencilla composición. La expresión de María es de un dramatismo muy contenido, el rictus de dolor no descompone la dulzura del rostro, y su pena aparece, solo visible, en la tristeza de la mirada.

La figura de Cristo descansa, blandamente recostada, sobre la pierna derecha doblada de María, al tiempo que extiende la izquierda para crear un hueco acogedor. Con una de las manos se ayuda para sostener la espalda y la cabeza, mientras que con la otra sostiene uno de los brazos. Cristo deja caer inerte el brazo derecho que llega a tocar el suelo y extiende ambas piernas formando, sin estridencias, un grupo piramidal. Su cuerpo, de suave anatomía, se cubre sumariamente con un paño de pureza, y muestra muy visible la sangre del costado. La cabeza, caída hacia un lado y ya sin corona, muestra barba y larga cabellera que se derrama por el costado derecho. Hilillos de sangre cruzan el rostro y muestra huellas de una herida muy significativa, la de una espina que ha entrado por encima de una de las cejas y ha salido sobre el párpado cerrado. La efigie presenta una novedad extremadamente curiosa, en el interior de

${ }^{14}$ Véase la obra de Javier Portús. Guía Velázquez. Museo del Prado. Madrid, 1999, pp. 188-189.

* Quiero expresar mi agradecimiento a Juan Félix Sánchez Sancho, joven investigador de la villa de Béjar, por su entusiasta colaboración para llevar a cabo este trabajo.

${ }^{1}$ Andrés Ordax, Salvador, «El escultor Alejandro Carnicero: su obra en Extremadura», Norba, 1980, pp. 9-25. Martín González, J.J., Escultura Barroca en España (1600 - 1770), Ed. Cátedra, Madrid, 1983. 